\title{
Réflexions d'un physicien sur les problèmes de formation
}

\section{Claude Garcin}

\section{(2) OpenEdition \\ 1 Journals}

Édition électronique

URL : https://journals.openedition.org/tc/1335

DOI : $10.4000 /$ tc. 1335

ISSN : 1952-420X

Éditeur

Éditions de l'EHESS

\section{Édition imprimée}

Date de publication : 1 juin 2005

ISSN : 0248-6016

\section{Référence électronique}

Claude Garcin, « Réflexions d'un physicien sur les problèmes de formation », Techniques \& Culture [En ligne], 45 | 2005, mis en ligne le 22 mai 2008, consulté le 29 septembre 2022. URL : http://

journals.openedition.org/tc/1335; DOI : https://doi.org/10.4000/tc.1335

Ce document a été généré automatiquement le 29 septembre 2022.

Tous droits réservés 


\title{
Réflexions d'un physicien sur les problèmes de formation
}

\author{
Claude Garcin
}

1 Considérations préliminaires

2 Pour toute société qui ne se désintéresse pas de son avenir, la formation est un enjeu majeur au même titre que la santé et les conditions d'existence.

3 Il s'agit là d'un constat que les historiens ont pu faire tout au long de l'histoire des communautés humaines et, dans la vie de nos sociétés actuelles, c'est un sujet récurrent de confrontation des points de vue et des enjeux sociaux et politiques, qu'il s'agisse de la formation initiale des jeunes ou de la formation des adultes au cours de leur vie professionnelle.

4 Les questions de formation constituent d'habitude le champ d'investigation des sciences de l'éducation, mais elles relèvent aussi des domaines étudiés par les ethnologues et, naturellement, par les historiens.

5 Ces questions ne font donc pas partie des champs d'investigation relevant de la compétence des physiciens en tant que scientifiques. En revanche, en tant que citoyens, les physiciens se doivent, au même titre que l'ensemble des scientifiques et que tous les autres citoyens, quel que soit leur domaine d'activité, de s'intéresser aux problèmes de la formation et d'y apporter leurs compétences propres, et ce parce que cela concerne le présent et l'avenir de la société.

6 Dans la communauté scientifique, les physiciens peuvent être caractérisés par le champ d'investigation qui est le leur -l'étude des propriétés générales de la matière et des lois qui gouvernent son comportement-, mais aussi par la culture qu'ils acquièrent dans l'exercice de leur activité professionnelle. À cet égard, ils sont porteurs d'une double culture :

7 - d'une part, une culture de l'observation qu'ils partagent avec l'ensemble des scientifiques, quelle que soit au demeurant leur discipline d'exercice ou leur spécialité ; 
8 - d'autre part, une culture de la modélisation qu'ils partagent avec un nombre plus restreint d'autres catégories de scientifiques, en particulier avec les mathématiciens et, plus largement, avec les théoriciens de toutes disciplines.

9 Ainsi, lorsqu'ils abordent d'une manière scientifique une question ne relevant pas de leur champ normal d'investigation, les physiciens le font naturellement avec cette double culture, tout en mettant en œuvre les deux processus méthodologiques fondamentaux que sont l'analyse et la synthèse.

10 À ce propos, arrêtons-nous sur le concept de culture, encore fortement marqué par la traditionnelle opposition entre la matière et l'esprit, et que l'on rattache d'ordinaire à l'esprit, c'est-à-dire à l'activité intellectuelle. En fait, une telle conception de la culture ne permet absolument pas de rendre compte des attitudes comportementales induites par les différentes activités humaines et sociales.

11 En vue de le rendre plus opératoire, le concept de culture doit être repensé et élargi de manière à pouvoir rendre compte de ces attitudes comportementales individuelles et sociales, lesquelles ne relèvent pas de l'inné dû à la transmission génétique, mais bien de l'acquis lié à la pratique et aux fréquentations et que l'on doit donc logiquement qualifier de culturelles.

12 À cet égard, il convient de préciser que l'habitude, dont la sagesse populaire affirme qu'elle est une seconde nature, se révèle être en fait un élément structurant de toute culture.

Transmission des connaissances et formation

13 Il convient également de s'arrêter sur le concept de transmission des connaissances, très largement utilisé, aussi bien par les pouvoirs publics que par de nombreux experts, et qui semble tellement bien aller de soi que l'on ne se donne même pas la peine de le soumettre à une réflexion critique et de vérifier la solidité de son fondement scientifique. Or, voilà bien un concept d'usage courant dont l'emploi ne peut se justifier qu'à la double condition, non explicitée en général :

14 - soit d'adopter le point de vue de Sirius, c'est-à-dire de se placer à une distance suffisante pour qu'il soit impossible d'entrevoir les mécanismes individuels constitutifs du processus de formation ;

15 - soit d'adopter le point de vue historique, c'est-à-dire de considérer des temps qui se situent à l'échelle de l'évolution des sociétés et, par conséquent, au minimum à celle du chevauchement des générations.

16 Les difficultés commencent dès lors que l'on veut appliquer un tel concept à la formation des individus, pour lesquels les mécanismes effectifs du processus de formation interviennent nécessairement, même si on ne les a pas clairement identifiés et caractérisés, et ce à une échelle de temps qui est toujours de l'ordre d'une fraction de génération ou, tout au plus, d'une génération.

$17 \mathrm{Au}$ demeurant, tout un chacun a pu constater au cours d'une période de scolarité ou de formation que, lorsque le cours ou l'exposé est terminé, rien n'est pour autant réglé en matière d'acquisition des connaissances, ce qui prouve à l'évidence qu'un processus de transmission ne peut, à lui seul, rendre compte du processus de formation lequel est en fait un processus plus complexe qu'il n'y paraît au premier abord.

18 De fait, un processus de transmission implique toujours, d'une part, un émetteur et un récepteur, d'autre part, un ou plusieurs mécanismes de transmission parfaitement 
caractérisable(s), et, enfin, un milieu et/ou un support de transmission. Et s'il y a bien en général un émetteur -l'enseignant ou le formateur- et un récepteur-l'apprenant, qu'il soit élève ou apprenti-, s'il y a bien des mécanismes de transmission, qu'ils soient visuels, vocaux ou gestuels, et, enfin, s'il y a bien un milieu de transmission plus ou moins sensible aux mécanismes de transmission, lequel milieu est dual et se décompose alors en un milieu ou support matériel et le milieu culturel ambiant-que ce dernier soit individuel ou social-, en revanche, il ne suffit pas qu'il y ait transmission effective des connaissances pour que l'acquisition de ces connaissances puisse être réalisée par l'apprenant.

Autrement dit, un processus de transmission ne peut en aucun cas rendre compte du processus d'acquisition des connaissances par les individus et, par voie de conséquence, le concept de transmission des connaissances se révèle inadapté pour parvenir à une représentation correcte du processus de formation à cette échelle.

$\mathrm{Au}$ plan méthodologique, lorsque la modélisation d'un processus ne donne pas satisfaction, il convient de recourir de nouveau à l'observation plus approfondie du processus considéré afin de mieux en caractériser le développement et mieux en identifier les différentes phases en vue de pouvoir en esquisser une nouvelle modélisation mieux adaptée à sa représentation. Une telle observation permet alors de se convaincre de ce que le message qui est transmis lors du processus d'enseignement ne correspond à des connaissances que pour celui qui le transmet -l'enseignant ou le formateur-alors que pour celui qui le reçoit -l'apprenant-, il ne correspond qu'à un ensemble d'informations structurées de manière cohérente en vue de leur assimilation ultérieure. Ce type d'observation permet également de se convaincre de ce que, une fois le processus d'enseignement terminé, commence un processus d'assimilation plus ou moins rapide, et que ce n'est qu'au terme du second processus que l'on peut enfin parler d'acquisition des connaissances.

21 Or, depuis les travaux de Piaget et de ses disciples sur l'acquisition de certains concepts fondamentaux par le jeune enfant, on sait que ce dernier se construit une représentation des phénomènes à partir de ce qu'il observe et qu'il peut éventuellement contrôler, et, plus généralement, à partir des informations vérifiables qu'il recueille ou qui lui sont fournies. En outre, les progrès des neurosciences au cours des dernières décennies ont validé les principales conclusions des études psychologiques que Piaget avait effectuées, et de plus, ils permettent d'induire que ce mode d'apprentissage par représentations interposées est transposable à toutes les étapes du développement de l'individu.

22 Ainsi, on peut raisonnablement penser que pour pouvoir comprendre les phénomènes ou les pratiques qu'il a besoin de maîtriser, chaque individu peut, à tout moment, s'en construire ou s'en reconstruire une représentation à partir de ce qu'il sait déjà et des informations complémentaires qui lui sont fournies ou qu'il peut éventuellement recueillir par lui-même.

De fait, quels qu'en soient la nature, la finalité et le niveau, toute formation se présente toujours comme un processus complexe :

24 - qui, en entrée, s'adresse à un public abordant la formation, lequel doit être clairement identifié et caractérisé, en fonction de la culture dont sont porteurs les individus qui le composent, compte tenu de leurs parcours antérieurs ; 
- et qui, en sortie, doit fournir des individus ayant acquis les capacités et compétences qualifiées requises par la société et/ou par le secteur d'activités à l'intention duquel a été configurée la formation concernée.

Après identification et caractérisation des objectifs de la formation, il convient donc de bien caractériser le processus global de formation lui-même ainsi que la relation pédagogique qui s'établit et se noue entre les acteurs-partenaires de ce processus.

Les acteurs-partenaires du processus global de formation sont naturellement, les enseignants et formateurs, d'une part, quels que soient la spécialité ou le secteur d'activités professionnelles dont ils relèvent ou dont ils proviennent, et les élèves, étudiants ou apprentis, d'autre part, quel que soit le type de formation qu'ils ont acquis antérieurement et le type de formation-initiale ou continue- qu'ils souhaitent acquérir.

À cet égard, il faut reconnaître, d'une part, que la relation qu'entretient un système structuré d'établissements de formation avec ses partenaires naturels (les pouvoirs publics et collectivités territoriales représentant la société à ses différents niveaux d'organisation, les organismes et entreprises participant aux divers secteurs d'activités économiques et sociales, les familles des élèves, apprentis ou étudiants concernés), est une relation de type fournisseur-client comme toute relation d'échange socioéconomique. D'autre part, que la relation pédagogique qui s'établit et se noue entre acteurs-partenaires du processus global de formation n'est pas une relation d'échange comme la relation d'échange économique, mais une relation complexe dont la structure est de nature complètement différente et qui peut être caractérisée comme une relation séquentielle ordonnée.

Essai d'analyse et de caractérisation du processus global de formation

En effet, alors que la modélisation du processus global de formation par un processus de transmission ne peut rendre compte de l'ensemble des problèmes que rencontrent tous les apprenants pour acquérir des connaissances, et que cette modélisation conduit, au plan institutionnel, à empiler les réformes en vue de colmater tant bien que mal les brèches qui apparaissent au fil du temps depuis que la formation revêt un caractère de masse, il devient impératif de repenser cette modélisation du processus de formation lui-même.

Ainsi, compte tenu des observations précédemment rappelées, il apparaît logique et possible d'envisager une modélisation du processus global de formation, non pas par un seul processus, mais par deux processus élémentaires consécutifs nécessairement articulés entre eux:

1 - un premier processus de réception des informations dans le cas de l'enseignement, ou de collecte des informations dans le cas d'une démarche autodidacte ;

- et un second processus, lui-même complexe, de traitement des informations recueillies, d'acquisition et d'appropriation des connaissances et des méthodes par la construction d'une représentation et, ensuite, par la vérification et la validation de cette représentation par sa mise en œuvre.

3 Naturellement, l'efficacité de chacun de ces deux processus élémentaires peut faire l'objet d'une évaluation, ainsi d'ailleurs que l'efficacité du processus global de formation qui, seul actuellement, y est soumis.

Le premier de ces processus élémentaires est, sous réserve de bien réaliser que la démarche autodidacte n'en constitue qu'un cas limite, principalement à la charge de l'enseignant ou 
$d u$ formateur: il présente un caractère plus ou moins collectif et correspond à une présentation des savoirs et savoir-faire et des méthodes afférentes.

Il faut ici noter la disproportion notoire existant la plupart du temps entre la présentation des méthodes d'élaboration des savoirs et savoir-faire dont l'importance est en général largement sous-estimée, alors même qu'elle est essentielle pour parvenir à une bonne formation méthodologique, et la présentation des méthodes d'utilisation des savoirs et savoir-faire, dont l'importance est en revanche largement surestimée, alors même qu'elle se relativise de plus en plus à une époque caractérisée par la rapidité d'évolution des technologies et le raccourcissement de leur durée de vie.

Enfin, il faut insister sur le fait que, contrairement à une idée pourtant largement répandue, ce processus élémentaire ne réalise pas par lui-même la formation, mais seulement la première partie de cette formation, celle qui se résume en dernière analyse à la présentation et à la réception ou à la collecte d'une information structurée de manière cohérente en vue de faciliter la réalisation du second processus élémentaire intervenant dans la formation.

Le deuxième de ces processus élémentaires est, quant à lui, essentiellement à la charge de l'élève, de l'étudiant ou de l'apprenti concerné. Il présente donc un caractère pratiquement individuel, même lorsqu'il s'accompagne d'échanges de points de vue et d'expériences plus ou moins collectives, et correspond à une transformation de l'information structurée de manière cohérente en connaissances proprement dites susceptibles de conférer à l'apprenant les capacités d'autonomie correspondantes, par l'appropriation et l'assimilation des savoirs et savoir-faire accompagnés de la maîtrise des méthodes afférentes. C'est le processus d'acquisition des connaissances et des méthodes ou processus d'apprentissage, précédemment évoqué, dont l'importance est essentielle, car en dernier ressort, c'est de lui que dépend la réussite ou l'échec du processus global de formation.

38 Si le premier des processus élémentaires qui intervient dans le processus global de formation est régulièrement enrichi par l'analyse des retours d'expériences effectuées par l'enseignant ou le formateur, le second processus ne fait pas - hormis le dispositif tutoral dans le cas des formations en alternance, couramment dénommées formations en apprentissage, relevant de la loi Astier- l'objet d'une organisation institutionnelle et d'un accompagnement pédagogique et méthodologique adapté systématique pour que l'élève, l'étudiant ou l'apprenti puisse mener à bien l'appropriation et l'assimilation des connaissances qui lui incombent et que personne ne peut effectuer à sa place.

Les progrès considérables de disciplines comme la psychologie -notamment avec les travaux de Piaget et depuis quelques décennies par les neurosciences-, mais aussi la linguistique et l'informatique -la construction de processus dits d'intelligence artificielle-, permettent aujourd'hui de mieux cerner ce que peut être le processus d'appropriation et d'assimilation des savoirs et savoir-faire et des méthodes afférentes ainsi que l'acquisition de la maîtrise correspondante. Ce processus, dont l'apprenant a la charge et la responsabilité, puisque nul autre ne peut se substituer à lui pour le mettre en œuvre et le conduire à son terme, est en fait un processus complexe de construction ou de reconstruction de son système de représentation de la réalité concernée, c'est-à-dire de ce que d'autres -enseignants et formateurs de toutes disciplines ou professionnels de tous secteurs d'activité- savent déjà et savent mettre en œuvre, chacun dans son domaine de compétences. considérer que tout individu est porteur, à chaque étape de son développement, d'un système 
de représentations de la réalité qui résulte des conceptions qu'il s'est forgé au cours de son parcours antérieur à partir :

41 - des savoirs et savoir-faire auxquels il a été confronté et des conceptions et représentations qu'il s'en est fait, ainsi que des idées reçues admises sans examen ;

42 - de ses expériences personnelles ainsi que, bien que dans une mesure tout à fait variable, de l'expérience de son environnement proche, lequel se transforme au cours de la vie en évoluant de l'environnement familial au stade de l'enfance jusqu'à l'environnement professionnel en principe à l'âge adulte, en passant par l'environnement très particulier des copains à l'âge de l'adolescence ;

43 - et enfin, de l'environnement médiatique et notamment télévisuel, lequel a pris une importance croissante au cours des dernières décennies.

44 Sous réserve d'un examen plus approfondi, on pourrait même ajouter que la construction d'une représentation repose, de manière plus ou moins explicite, sur un double processus élémentaire d'analyse des informations et, ensuite, de synthèse des données acquises conduisant à une modélisation dont la représentation ne serait que l'image. Ce serait donc du caractère opératoire de cette modélisation et de sa représentation que dépendrait alors l'efficacité de sa mise en œuvre.

45 Par voie de conséquence, la présentation des connaissances nouvelles-savoirs et savoir-faire- et des méthodes afférentes implique une confrontation de cette information structurée de manière cohérente qui a été fournie à l'apprenant par le maitre, ou qu'il est allé rechercher lui-même dans le cas limite d'une démarche autodidacte, avec le système de représentations dont il est porteur.

Plus précisément, avant même de pouvoir être réalisée, la construction d'une représentation fait nécessairement intervenir une confrontation logique de compatibilité entre la cohérence de la représentation antérieure fondée sur les savoirs déjà acquis et la cohérence impliquée par les informations nouvelles qui peuvent bousculer plus ou moins, voire briser la cohérence de la représentation antérieure en révélant des incompatibilités plus ou moins grandes avec cette cohérence. Il faut donc examiner les deux cas limites d'une telle compatibilité des cohérences.

47 Le premier cas limite est celui où les informations nouvelles sont totalement compatibles avec la cohérence de la représentation associée aux savoirs acquis antérieurement: il s'agit alors de compléter une représentation déjà partiellement réalisée, de la même manière que l'on poursuit la réalisation d'un puzzle avec des pièces nouvelles. Ce qui, au demeurant, n'enlève rien à la difficulté propre d'une telle opération pour l'apprenant concerné !...

48 L'autre cas limite est celui où les informations nouvelles sont totalement incompatibles avec la cohérence de la représentation associée aux savoirs acquis antérieurement : on se trouve alors dans une situation analogue, au plan psychologique, à celle que connaissent les médecins, au plan physiologique, avec le rejet de la greffe d'organes. Mais si les médecins peuvent tenter de neutraliser les facteurs de rejet pour réussir une greffe, les formateurs ne peuvent avoir recours à une telle méthode et ils se doivent -si toutefois on veut bien leur en laisser le loisir et le temps- d'aider les apprenants à se construire une nouvelle représentation dont la cohérence, plus générale, soit compatible avec celle impliquée par les informations nouvelles, tout en demeurant compatible avec celle de la représentation associée aux savoirs acquis antérieurement. 

que la traduction du langage parlé dont le socle est en partie déjà acquis, le jeune enfant doit affronter les difficultés inhérentes au passage d'une cohérence souple et souvent fluctuante à une cohérence beaucoup plus rigide dont les règles peuvent se révéler très contraignantes au premier abord. Après l'acquisition de l'alphabet, la représentation écrite des sons et la lecture des syllabes sont très vraisemblablement les clefs de la compréhension et de l'assimilation de la correspondance entre le langage écrit et le langage parlé (ou le langage des gestes pour les sourds-muets), dont la maîtrise requiert assurément une longue pratique des deux processus complémentaires et réciproques que sont l'écriture et la lecture.

Il en va de même lors de l'acquisition de la représentation permettant le traitement des grandeurs orientées, lorsque le collégien doit affronter les difficultés inhérentes au passage d'une cohérence rigide simple à une cohérence rigide plus complexe dont les règles peuvent se révéler déroutantes. Après l'acquisition de l'outil mathématique usuel permettant la représentation et le traitement des grandeurs non orientées encore dénommées grandeurs scalaires-, telles que longueurs, durées ou températures, la représentation et le traitement des grandeurs orientées, telles que vitesses ou forces, nécessite l'élaboration de nouveaux outils mathématiques, plus complexes, qui permettent de parvenir à une représentation correcte et à un traitement opérationnel des grandeurs orientées incluant, pour toute orientation considérée, la représentation et le traitement des grandeurs scalaires associées.

51 Et l'on pourrait d'ailleurs procéder à des analyses comparables lorsque, en Physique, on doit passer de la mécanique terrestre, avec frottements et forte pesanteur, à la mécanique spatiale, quasiment sans frottements et avec une pesanteur très faible voire nulle, ou de la mécanique classique des objets usuels ayant des vitesses faibles ou moyennes à la mécanique des particules dites relativistes parce qu'ayant de très grandes vitesses plus ou moins proches de la vitesse de la lumière ou, enfin, lorsqu'on doit passer de la physique classique adaptée aux objets macroscopiques à la physique quantique adaptée aux atomes, aux électrons et aux particules dites élémentaires.

Il est tout à fait raisonnable de penser que l'on peut trouver dans chaque domaine d'activités des ruptures de cohérence qui, à un moment donné, exigent une remise en question du système antérieur de représentation et l'élaboration corrélative d'un nouveau système de représentation susceptible d'intégrer les connaissances nouvelles et les savoirs antérieurement acquis. Il en va ainsi, lorsque, par exemple, l'ouvrier ou le technicien doit passer de la commande manuelle d'une machine-outil ou d'un appareil à sa commande numérique et, ultérieurement, à sa commande informatique pilotée par ordinateur.

Pour l'apprenant, confronté à son travail d'apprentissage en l'absence de toute rupture de cohérence entre connaissances nouvelles et savoirs acquis, le travail élémentaire en vue de compléter une représentation déjà partiellement réalisée consiste à situer les connaissances nouvelles par rapport à celles acquises antérieurement, à les « emboîter » ensemble de manière à ce qu'elles s'articulent logiquement et à vérifier la validité opéra-tionnelle de cette représentation ainsi complétée. Un tel travail d'appren-tissage ne peut être laissé à l'abandon d'une progression aléatoire par essaiserreurs, au demeurant fastidieuse et vite lassante, et requiert impé-rativement, pour être efficace, un réel accompagnement pédagogique et méthodologique approprié.

Techniques \& Culture, 45 | 2005 
54 Il est inutile d'insister sur le fait que, lorsqu'elle s'avère nécessaire, la construction d'une représentation nouvelle, qui ne peut se résoudre à une simple greffe, exige en fait la déconstruction logique de la représentation antérieure et l'analyse méthodologique de sa cohérence et des contradictions qu'engendrent les connaissances nouvelles; après quoi, la construction d'une représentation nouvelle requiert l'élaboration d'une cohérence, plus générale et plus complexe, susceptible d'englober la cohérence exigée par les connaissances nouvelles et celle correspondant aux savoirs acquis antérieurement et, par voie de conséquence, l'articulation logique de ces cohérences, requiert naturellement un accompagnement pédagogique et méthodologique renforcé, cela va sans dire.

55 Faute d'un tel accompagnement méthodologique approprié, dûment pensé et organisé, c'est-à-dire en situation d'abandon pédagogique, l'apprenant se retrouve seul et livré à lui-même pour effectuer un travail d'apprentissage que nul ne peut faire à sa place, et il ne lui reste guère que le recours à la mémoire pour tenter de s'approprier tant bien que mal les connaissances nouvelles en mémorisant les concepts et les symboles ainsi que les opérations et les gestes. À cet égard, deux cas extrêmes peuvent se présenter avec, bien entendu, tous les cas intermédiaires possibles :

56 - soit l'apprenant possède l'atout de disposer d'une bonne mémoire auquel cas il va utiliser au mieux cette mémoire, faute de pouvoir se construire cette nouvelle représentation permettant de réaliser une intégration des connaissances nouvelles et des savoirs acquis; il va alors, comme cela se pratique couramment en informatique, ouvrir un nouveau fichier de mémorisation et, au fil du temps, autant de fichiers de mémorisation qu'il sera nécessaire en se rappelant que, pour tel problème, il faudra s'adresser à tel fichier et, pour tel autre problème, à tel autre fichier, une telle stratégie lui assurant, sinon une bonne compréhension, à tout le moins la possibilité de s'en sortir tout en faisant l'économie de la reconstruction d'une représentation adaptée à une réalité plus complexe englobant connaissances acquises antérieurement et connaissances nouvelles ;

57 - soit l'apprenant ne possède qu'une mauvaise mémoire, auquel cas il va devoir galérer pour en arriver, dans le pire des cas, à une attitude de retrait progressif se terminant par un abandon pur et simple, ou à une attitude de révolte et de rejet systématique pouvant déboucher, à la période de l'adolescence, sur une violence scolaire plus ou moins ouverte et pouvant, à la limite, se conjuguer avec les autres violences inhérentes à la société ambiante.

Essai préliminaire d'identification et de caractérisation des conséquences logiques de l'analyse du processus global de formation

Devant les conséquences constatées de la massification de la formation initiale, et sous l'empire des nécessités, la réflexion institutionnelle n'a pris en compte, jusqu'à présent, que la réalité de l'importance de l'échec scolaire et l'obligation d'organiser la mise en œuvre d'un soutien scolaire, notamment en cas de grandes difficultés rencontrées par les élèves pendant la période de scolarité obligatoire, et ce, sans jamais s'interroger vraiment sur la nature même du processus global de formation et, par voie de conséquence, sur les conditions nécessaires à sa réussite.

59 Or si, toutes vérifications nécessaires effectuées, l'analyse du processus global de formation est confirmée, il faudra bien alors en tirer toutes les conséquences logiques. 
60 D'une part, il faudra alors redéfinir l'activité et le service des enseignants afin d'y inclure obligatoirement l'accompagnement pédagogique et méthodologique des élèves, ce qui implique une forte augmentation de leur nombre pour assurer la prise en charge de cet accompagnement.

61 D'autre part, et corrélativement, il faudra alors redéfinir la formation des enseignants et des formateurs afin de les préparer à cette fonction d'accompagnement pédagogique et méthodologique des élèves dans la mise en œuvre du processus d'appropriation et d'assimilation des connaissances et des méthodes et, en particulier, donner aux futurs enseignants et formateurs une formation méthodologique et pratique adéquates, ce qui pourrait être efficacement réalisé dans le cadre d'une formation en alternance.

De fait, les implications socio-économiques d'un tel changement de paradigme de la formation s'avèrent considérables à l'horizon de sa mise en œuvre. Il s'agit là d'un véritable problème de société auquel il faut se confronter. C'est à la société qu'il incombe de décider -et cela nécessite un large débat public-, car il s'agit de son propre avenir, immédiat à travers la réorganisation nécessaire de la formation permanente des adultes et, à court terme, à travers la non moins nécessaire réorganisation de la formation initiale des jeunes.

\section{RÉSUMÉS}

Lorsqu'un physicien sort de son champ de compétences pour s'aventurer dans le domaine de la formation, il constate que, sauf exception, la formation des individus repose essentiellement sur la mise en œuvre du concept de transmission des connaissances, lequel se révèle tout à fait inadapté à une description correcte du processus de formation à cette échelle. S'il tente d'effectuer une nouvelle modélisation du processus de formation à l'échelle des individus, il est conduit à considérer que ce processus se décomposera en fait en deux processus élémentaires consécutifs : un premier processus de réception ou de collecte des informations, correspondant à des connaissances; un second processus de traitement des informations recueillies et de construction mentale d'une représentation de ces connaissances, suivie d'une validation de cette représentation par sa mise en œuvre. Il faut ensuite examiner les principales conséquences logiques de cette nouvelle modélisation du processus de formation.

\section{A physician's reflections about the problems of teaching.}

When a physician gets out his field to explore the field of education, he sees that teaching is most exclusively based on the concept of transmitting knowledge. This concept proves to be quite unsuitable to describe correctly the individual level in the educational process. When the physician tries to make a new modelisation of this process, he is led to consider that it has to be divided in two elemantary and consecutive processes : a first process of reception or gathering of information, linked to knowledge ; a second one treating the collected information, and building a mental construction of a representation of this knowledge. A validation through practice follows this mental construction. We must then analyse the main logical results ot this new modelisation of the process of teaching. 
Reflexiones de une físico acerca de los problemas de formación.

Cada vez que un físico sale de su terreno de pericia para aventurarse en el campo de la formación, se da cuenta de que, salvo exepción, la formación de los individuos se apoya por lo esencial en el concepto de trasmisión de los saberes. Este se revela totalmente impropio para describir el proceso de formación según parecida escala. Si dicho físico intenta elaborar una nueva modelización del proceso de formación a escala de individuos, tiene que considerar la división de ese proceso en dos procesos elementales consecutivos : el primero, de recepción o de recolección de las informaciones, que corresponde a saberes; el segundo, de tratamiento de las infomación regogidas y de construcción mental de la representación de estos saberes, esta última siendo seguida por una validación de dicha representación mediante su aplicación. Después, es preciso examinar las principales conscuencias lógicas de esta nueva modelización del proceso de formación.

INDEX

Mots-clés : représentations, modélisation, connaissances

Keywords : representations, education, knowledge, modelisation, formation

\section{AUTEUR}

\section{CLAUDE GARCIN}

Professeur honoraire de Physique à l'Université Pierre et Marie Curie - Paris 6. 